\title{
Verfassungs- und strafvollzugsrechtliche Aspekte der Privatisierung im Strafvollzug
}

Heinz Müller-Dietz

\section{Verfassungsrechtliche Aspekte der Privatisierung im Strafvollzug}

Der seit über einem Jahrzehnt andauernde Diskurs über die Möglichkeiten, die das GG einer Privatisierung eröffnet, und die Schranken, die sie ihr zieht, hat zwar in grundsätzlicher Hinsicht zu einer weitgehenden Übereinstimmung geführt. Eine restlose Einigung namentlich über die Grenzen einer Privatisierung hat er bisher indessen nicht gebracht ${ }^{1}$. Das hat verschiedene Gründe, die hier nur stichwortartig angedeutet werden können. Zum einen sind natürlich auch die Verfassung, ihre Auslegung und Handhabung einem Wandel unterworfen, der durch die gesellschaftliche und wirtschaftliche Entwicklung keineswegs unberührt bleibt. Zum anderen kann man eine wachsende Ökonomisierung aller Lebenslagen und -verhältnisse konstatieren ${ }^{2}$, die auch die auf den Feldern der Kriminalprävention und »inneren Sicherheit« agierenden Institutionen in ihren Bann gezogen, wenn nicht heimgesucht und dort ihre unübersehbaren Spuren hinterlassen hat ${ }^{3}$.

Weitgehende Einigkeit besteht in der Auffassung, dass eine Privatisierung des Strafvollzugs im Sinne einer Übertragung der dem Staat auf diesem Gebiet obliegenden öffentlichen Aufgaben - etwa nach dem Muster Großbritanniens und der USA ${ }^{4}$ mit der Verfassung unvereinbar ist ${ }^{5}$. Die Vollziehung der Freiheitsstrafe zählt zu den Kernaufgaben hoheitlicher Verwaltung. Sie steht daher als Ausdruck des staatlichen Gewaltmonopols unter dem Funktionsvorbehalt des Art. 33 IV GG. ${ }^{6}$. Die damit verbundenen Eingriffe in Grundrechte des Gefangenen erfordern daher in aller Regel die Wahrnehmung der verfassungsrechtlichen Aufgaben, die dem Staat insoweit obliegen ${ }^{7}$, durch Beamte, die in einem ständigen Dienst- und Treueverhältnis zum Staat stehen.

Diesen Funktionsvorbehalt bringt § 155 I 1 StVollzG zum Ausdruck. Danach sind die Aufgaben des Strafvollzugs von Beamten wahrzunehmen. Eine Beleihung privater Unternehmen in Form einer Organisations-, Funktional- oder Verfahrensprivatisierung ist damit verfassungsrechtlich unzulässig ${ }^{8}$. Was hiernach als Möglichkeit der Mitwirkung Privater übrig bleibt, ist die Übernahme von Service-Funktionen im Wege der Verwaltungshilfe.

Freilich ist diese Auffassung keineswegs unangefochten. Eine Mindermeinung, die sich allerdings nicht durchzusetzen vermocht hat, geht da- von aus, dass weder das Rechtsstaats- und das Demokratieprinzip des GG noch die Grundrechte des Gefangenen der Schaffung von privat geführten Vollzugsanstalten entgegensteht ${ }^{9}$. Ihr zufolge wäre es verfassungsrechtlich zulässig, nach anglo-amerikanischem Muster neben staatlichen Gefängnissen auch private $\mathrm{zu}$ errichten und zu betreiben, die dann natürlich unter staatlicher Aufsicht stehen. Diese Meinung, die das staatliche Gewaltmonopol und damit die verfassungsrechtliche Stellung des Staates im Verhältnis zu seinen Bürgern verkennt, hat indessen zu Recht keinen Anklang gefunden.

Demgegenüber haben Auffassungen in Theorie und Praxis an Boden gewonnen, die an die »klassische« Unterscheidung von Eingriffs- und Leistungsverwaltung anknüpfen und daraus - wenn auch in durchaus unterschiedlicher Weise - differenzierende Lösungen auf dem Gebiet der Privatisierung im Strafvollzug herleiten. Hiernach sind grundrechtsbeschränkende Maßnahmen, die im Wege der Eingriffsverwaltung vorgenommen werden, auf Grund des Gewaltmonopols Beamten vorbehalten; sie sind dementsprechend einer Privatisierung nicht zugänglich. Demgegenüber soll nach dieser Ansicht die Erfüllung von Vollzugsaufgaben im Wege der Leistungsverwaltung sehr wohl Privaten übertragen werden können. Das hat der Sache nach zur Folge, dass Behandlungsaufgaben, die der sozialen Integration des Gefangenen dienen, ganz oder teilweise privatisiert werden können ${ }^{10}$.

Diese Auffassung fußt offensichtlich auf einer Aufspaltung der Aufgaben des Strafvollzugs, die den verfassungsrechtlichen Vorgaben - wie sie in der Rechtsprechung des Bundesverfassungsgerichts grundgelegt sind ${ }^{11}$ - zuwiderläuft. Danach sind die in $\S 2$ StVollzG festgeschriebenen Aufgaben der Resozialisierung des Gefangenen und des Schutzes der Allgemeinheit gleichermaßen hoheitlicher Natur, so dass ihre Wahrnehmung dem Staat obliegt. Sie werden daher beide vom Funktionsvorbehalt des Art. 33 IV GG erfasst ${ }^{12}$. Damit gehören nicht nur Grundrechtseingriffe (qua Eingriffsverwaltung) zu den hoheitlichen Aufgaben des Staates, sondern auch die grundrechtswesentlichen Leistungen, die er im Hinblick auf die Resozialisierung der Gefangenen zu erbringen hat. Die Vorenthaltung solcher Leistungen, die dem verfassungsrechtlichen Anspruch des Gefangenen auf Resozialisierung entsprechen ${ }^{13}$, kann ihn einschneidender treffen als ein »klassischer « Eingriff in seine Grundrechte. Dementsprechend ist der Behandlungsauf- trag grundsätzlich - mit den skizzierten Einschränkungen - ebenso wie die Sicherungsaufgabe von staatlichen Bediensteten wahrzunehmen.

Durchbrechungen dieser Grundsätze lassen sich auch nicht mit fiskalischen Erwägungen rechtfertigen ${ }^{14}$. Überdies steht auch der empirische Nachweis, dass private Unternehmen kostengünstiger, rationeller und - im Hinblick auf die Vollzugsaufgaben - effektiver arbeiten, bisher nach wie vor aus ${ }^{15}$. Dies gilt jedenfalls insoweit, als sich die Justizvollzugsanstalten neuer Organisations- und Betriebsformen und Steuerungsinstrumente bedienen, wie sie in erfolgversprechender Weise in der freien Wirtschaft praktiziert werden ${ }^{16}$.

Der enge sachliche Zusammenhang zwischen Resozialisierungsziel und Sicherungsaufgabe untermauert obendrein diese Sichtweise. Das zeigt sich namentlich bei Behandlungsmaßnahmen wie Vollzugslockerungen und Hafturlaub, deren Gewährung ja u. a. unter dem Vorbehalt der Missbrauchsgefahr (§ 11 II StVollzG9 steht ${ }^{17}$. Zudem hat das Bundesverfassungsgericht ausgesprochen, dass eine auf soziale Integration ausgerichtete Ausgestaltung des Strafvollzugs sich mitnichten in bloßen Angeboten und Leistungen erschöpft. Vielmehr können demzufolge auch grundrechtsbeschränkende Maßnahmen erforderlich werden, »um die inneren Voraussetzungen für eine spätere straffreie Lebensführung des Gefangenen zu fördern ${ }^{18}$.

Hier spielt auch eine wesentliche Rolle, dass es de lege lata keine Vollzugsbediensteten mehr gibt, die lediglich Aufgaben der Überwachung und Kontrolle wahrzunehmen haben ${ }^{19}$. Es ist gerade aus guten Gründen die Intention des StVollzG, an der Erfüllung der Vollzugsaufgaben alle Dienste und Mitarbeiter der Vollzugsanstalt - wenn auch natürlich in differenzierter und abgestufter Weise - zu beteiligen. Würden hingegen Behandlungsmaßnahmen - wie etwa Ausbildung und Therapie - in wesentlichem Umfange auf Mitarbeiter privater Unternehmen übertragen, dann würde namentlich der Allgemeine Vollzugsdienst auf den Status des Aufsichtsdienstes früherer Prägung zurückgeworfen. Er bliebe in der Hauptsache auf kontrollierende und disziplinierende Funktionen beschränkt, was sowohl dem Resozialisierungskonzept des StVollzG als auch dem Selbstverständnis und Berufsbild dieses Dienstes zuwiderliefe. 


\section{Die Ausnahme des § 155 I 2 StVollzG}

Allerdings sieht $§ 155$ I 2 StVollzG eine Ausnahme von dem Grundsatz vor, dass die hoheitlichen Aufgaben des Strafvollzugs von Beamten wahrgenommen werden müssen. Von dieser Befugnis darf indessen nur bei Vorliegen bestimmter persönlicher und sachlicher Voraussetzungen Gebrauch gemacht werden. So darf mit der Wahrnehmung solcher Aufgaben nur ein eigens rechtlich Verpflichteter Personenkreis betraut werden. Ferner darf die Aufgabenübertragung nur erfolgen, wenn dafür besondere sachliche Gründe gegeben sind.

Neben anderen Bediensteten der Justizvollzugsanstalten zählen zu dem in $§ 155$ I 2 genannten Personenkreis nebenamtlich oder vertraglich verpflichtete Personen. Nebenamtlich verpflichtete Personen sind in anderen Bereichen des öffentlichen Dienstes in hauptamtlicher Funktion tätig. Ihnen kann zusätzlich die Wahrnehmung nebenamtlicher Tätigkeiten im Strafvollzug übertragen werden. Einen solchen Fall regelt etwa § 158 I 2 StVollzG, der es bei Vorliegen besonderer Gründe gestattet, von der Regel abzuweichen, wonach die medizinische Versorgung der Gefangene durch hauptamtliche Ärzte sicherzustellen ist. Demgegenüber handelt es sich bei vertraglich verpflichteten Personen um Private, die neben ihrer eigentlichen beruflichen Tätigkeit bei der Erfüllung hoheitlicher Aufgaben des Strafvollzugs als Verwaltungshelfer hinzugezogen werden können. Sie nehmen dann im Auftrag und nach Maßgabe ihrer vertraglichen Verpflichtungen für die Vollzugsbehörde untergeordnete Funktionen wahr, die in aller Regel unterstützenden oder Servicecharakter haben ${ }^{20}$.

Besondere Gründe i.S. des § 155 I 2 StVollzG liegen nur vor, wenn die Hinzuziehung nebenamtlich verpflichteter Personen oder privater Verwaltungshelfer sachlich geboten und - im Hinblick auf den Ausnahmecharakter der Regelung - auch vertretbar ist. Notwendig kann etwa die Mitwirkung externer Fachkräfte dann werden, wenn sie nur von Fall zu Fall tätig werden müssen, so dass sich eine entsprechende personelle Ausstattung der Vollzugsanstalt nicht lohnt ${ }^{21}$. Dagegen gestattet es § 155 I 2 keineswegs, etwaige personelle Engpässe oder Notlagen durch Heranziehung von Mitarbeitern privater Unternehmen zu beheben. Denn nach § 155 II StVollzG ist es ja Sache des Staates, für eine hinreichende personelle Ausstattung der Vollzugsanstalten zu sorgen. Er darf sich dieser Verpflichtung nicht durch die Inanspruchnahme Privater entziehen, welche die Beamten obliegenden Aufgaben wahrnehmen sollen. Dementsprechend deckt die Vorschrift auch eine Handhabung nicht, die einen erheblichen - etwa $30 \%$ - übersteigenden Anteil von privaten Mitarbeitern bei der Erfüllung der einer Vollzugsanstalt obliegenden Aufgaben zur Voraussetzung hat ${ }^{22}$.

\section{Entwicklungstendenzen in der Straf- vollzugspraxis}

Indessen ist die Entwicklung mancherorts längst über die hier dargelegten verfassungs- und strafvollzugsrechtlichen Schranken der Privatisierung hinweggegangen. Dies gilt zum einen für die Übertragung von Behandlungsaufgaben auf Private ${ }^{23}$, zum anderen für die Privatisierung von staatlichen Eigenbetrieben, die bisher der Anstaltsversorgung gedient haben ${ }^{24}$. Beides hat - wenn auch aus je verschiedenen Gründen - gewichtige Konsequenzen für die Erfüllung der hoheitlichen Aufgaben, die dem Strafvollzug - keineswegs nur gegenüber Strafgefangenen, sondern auch der Allgemeinheit obliegen. So bedeutsam die Öffnung der Anstalten im Hinblick auf die Teilhabe der Gesellschaft auch in Gestalt externer Mitarbeiter privater Unternehmen - ist ${ }^{25}$, so muss es eben wegen des hoheitlichen Charakters der Vollzugsaufgaben dabei bleiben, dass die Justizvollzugsanstalten in der Substanz dem Resozialisierungsauftrag in eigener Regie nachkommen und sich nicht im Wesentlichen auf die Wahrnehmung von Sicherungsund Kontrollfunktionen beschränken. Wird die Versorgung der Justizvollzugsanstalt - die bisher in Gestalt von Eigenbetrieben (wie Küche, Bäckerei, Wäscherei, Gärtnerei usw.) wahrgenommen worden ist - weitgehend oder ganz in private Hände gelegt, droht die Gefahr, dass die dort vorhandenen Arbeits- und Ausbildungsplätze für Gefangene verloren gehen ${ }^{26}$. Die Anstalten sind aber, wie dargelegt, im Hinblick auf ihren Resozialisierungsauftrag gerade dazu verpflichtet, optimale Bedingungen für die Beschäftigung und die (berufliche) Ausbildung der Gefangenen zu gewährleisten. Diese Verpflichtung kann nicht dadurch substituiert werden, dass sich private Unternehmen vertraglich dazu verpflichten, Gefangene in von ihnen geführten Versorgungsbetrieben zu beschäftigen und auszubilden. Denn auch in einem solchen Falle begäbe sich die Justizvollzugsanstalt - ungeachtet der ihr verbleibenden Leitungsgewalt und Aufsichtsbefugnisse einer wichtigen Möglichkeit zur eigenständigen Gestaltung und Steuerung des Arbeits- und Ausbildungsangebots und zur inhaltlichen Ausgestaltung und Handhabung des für den einzelnen Gefangenen zu erstellenden Vollzugsplans (§ 7 StVollzG).

Ob auch Mitarbeiter privater Unternehmen den inzwischen deutlich gestiegenen Anforderungen an einen person- und sachgerechten Umgang mit Insassen gewachsen wären, ist eine zumindest offene Frage. Natürlich werden sie durch Teilnahme an einschlägigen Kursen oder anderen Veranstaltungen auf ihre neuen Aufgaben vorbereitet, schon weil die privaten Anbieter vertraglich gehalten sind, geeignetes Personal zur Verfügung zu stellen. Doch kann man schon gelinde Zweifel daran hegen, dass die strengen Maßstäbe, welche die Landesjustizverwaltungen an die Auswahl und Ausbildung ihrer Mitarbeiter anlegen, entsprechende Standards auf der privaten Seite zur Folge haben. Das ist vielfach schon von der Vertrautheit mit der besonderen Materie des Strafvollzugs und den dort bestehenden Rahmenbedingungen her nur schwer zu gewährleisten. Ganz abgesehen davon reicht die Vorbereitung privater Anbieter auf den Tätigkeitsbereich Strafvollzug bereits in zeitlicher Hinsicht nicht an die einschlägige Ausbildung der Anwärter in den Justizvollzugsschulen heran. Insoweit kann die in Absage an Privatisierungstendenzen anlässlich des 25-jährigen Bestehens der bayerischen Justizvollzugsschule formulierte Feststellung der Justizministerin nur unterstrichen werden: »Es gibt keinen Verzicht auf motivierte Mitarbeit. Nur gut qualifiziertes Personal kann mit problematischen Gefangenen richtig umgehen. " $27 \mathrm{Zu}$ befürchten ist freilich, dass die von Wissenschaft und Praxis vergeblich kritisierte ${ }^{28}$ Übertragung der Gesetzgebungszuständigkeit für den Strafvollzug vom Bund auf die Länder ${ }^{29}$ weitergehenden Privatisierungsbestrebungen neuen, zusätzlichen Auftrieb verleihen wird ${ }^{30}$.

\section{Zur heutigen Problematik der Beschäf- tigung und (Aus-)Bildung Gefangener}

Die Frage der Beschäftigung und (beruflichen) Bildung Gefangener gewinnt angesichts der allgemeinen gesellschaftlichen Situation und Entwicklung auf dem Arbeitsmarkt und in der freien Wirtschaft unter den Vorzeichen wachsenden Konkurrenzdrucks und der Globalisierung zunehmend an Bedeutung. Was der Strafvollzug im Bereich der Beschäftigung Gefangener - oder vielmehr des Verlustes an Arbeitsplätzen - gegenwärtig erlebt, hat demnach tiefere und weiter reichende Ursachen, als sie die Probleme häufig verkürzende Engführung des Themas herauszuarbeiten vermag. Wenn aber der Blick für Zusammenhänge fehlt oder verloren geht, dann zieht das notwendigerweise auch strukturelle Defizite in den Lösungsansätzen nach sich.

Die bisherige Diskussion über (Teil-)Privatisierung im Strafvollzug leidet vielfach unter einem immer wiederkehrenden vollzugsspezifischen Mangel: nämlich der häufigen Beschränkung auf den engeren, die Vollzugsanstalten unmittelbar betreffenden Fragenkreis, welche Dienstleistungen unter verfassungs-, strafvollzugs- und haushaltsrechtlichen sowie ökonomischen Aspekten für die Wahrnehmung durch Unternehmen der freien Wirtschaft in Betracht kommen. Ausgeblendet werden dabei oft genug folgenreiche innere Zusammenhänge mit gesamtgesellschaftlichen Entwicklungen - und damit deren Auswirkungen auf den Strafvollzug und dessen Handlungsmöglichkeiten. Das gilt vor allem für den Wandel der Industrie- zur Dienstleistungs- und Informationsgesellschaft, die fortschreitende Technisierung und Rationalisierung der Arbeitsabläufe, Produktionsverhältnisse und vorgänge sowie - in diesem Kontext - für die überaus schwierig gewordene Lage auf dem Arbeitsmarkt. Wobei der Begriff »Dienstleistungsgesellschaft « angesichts zunehmender Verdrängung von Menschen durch Apparate einen eigentümlich unpersönlichen Inhalt erhalten hat. 
Zwar wird allenthalben registriert, in welchem Maße auch die Justizvollzugsanstalten und ihre Arbeitsbetriebe - erst recht aber die Gefangenen selbst - von diesen Veränderungsprozessen, insbesondere von der allmählich chronisch erscheinenden Arbeitslosigkeit, betroffen sind ${ }^{31}$. Einschlägige Analysen verweisen auf den außerhalb der Mauern bestehenden Kostendruck - der natürlich an die Anstalten weitergegeben wird -, auf die Konkurrenz von Billiglohnländern - die es zuweilen sogar schafft, ohnehin günstige Angebote von Arbeitsbetrieben noch zu unterbieten -, auf die Rationalisierungseffekte - die in zunehmender Weise Maschinen an die Stelle menschlicher Arbeitskraft treten lassen.

Zwar werden im Strafvollzug längst Ansätze der Gegensteuerung praktiziert - etwa Modelle der freien Wirtschaft im Arbeitsbetriebswesen realisiert, kostensparende Formen der Organisation, Verwaltung und Abrechnung angewandt, neue Produkte entwickelt und neue Märkte erschlossen, die Werbung heutigen Anforderungen angepasst ${ }^{32}$. In der Tat haben die Beteiligung am wirtschaftlichen Wettbewerb sowie die Nutzung heutiger technischer, insbesondere elektronischer Möglichkeiten der Produktion und der Werbung dazu beizutragen vermocht, Arbeitsplätze hinter Mauern zu sichern, wenn nicht gar neu zu schaffen. Jedoch ist es auch in diesem Bereich bei der Grunderfahrung geblieben, dass der Strafvollzug auf generelle ökonomische und soziale Probleme, die ihm selbst vorausliegen, nur mit Konzepten begrenzter Reichweite reagieren, nachteilige Folgen gesellschaftlicher Probleme und Konflikte nur abmildern kann. Die Grundproblematik selbst kann er natürlich nicht aus der Welt schaffen.

Die Anzeichen dafür, dass bezahlte - und unter Gewinnaspekten bezahlbare - Arbeit auf die Dauer knapp wird, sind bereits spätestens gegen Ende des 20. Jahrhunderts sichtbar geworden. Es sind wohl eher Sozialwissenschaftler als Ökonomen gewesen, die damals das »Ende der Arbeitsgesellschaft«, wenn nicht diagnostiziert, so doch prognostiziert haben ${ }^{33}$. Anders sind ja die Konzepte schwerlich zu verstehen, die - wie etwa das Modell, für die Allgemeinheit sinnvolle, ja notwendige »Bürgerarbeit« aus Steuermitteln zu finanzieren größeren gesellschaftlichen Gruppen die sonst auf Dauer drohende Arbeitslosigkeit ersparen sollen. Sie fußen auf der Annahme, dass auf Grund fortschreitender Technisierung und Rationalisierung die ja längst auch den Markt der Dienstleistungen einbeziehen - mit einem zunehmenden Rückgang an Arbeitsplätzen zu rechnen ist. Natürlich hat der Verlust an Arbeitsplätzen - der häufig als Folge von Konzentrations- und Fusionstendenzen in der Wirtschaft eintritt - auch noch andere Ursachen, die z. B. in den ökonomischen Möglichkeiten liegen, welche die Börse Aktionären und Spekulationsgewinnen eröffnet. Dass solche globalen Phänomene, die gewichtige Auswirkungen auf den Arbeitsmarkt haben, weder nationale Regierungen noch internationale Institutionen bis- her hinreichend in den Griff bekommen haben, zeigt die Dimension der Probleme an, vor denen insoweit ganze Gesellschaften stehen.

Wenn solche Diagnosen oder Prognosen auch nur ansatzweise die wirtschaftliche und Arbeitsmarktentwicklung zutreffend beschreiben, dann erweisen sich die landläufigen ökonomischen »Rezepte«, die auf mehr Wachstum der Wirtschaft setzen und davon eine fühlbare Verbesserung der Situation auf dem Arbeitsmarkt erwarten, als problematisch. In diesem Sinne ist denn auch kürzlich »die Schulweisheit ,Mehr Wachstum gleich mehr Arbeitsplätze' « als schlechte Ideologie kritisiert worden ${ }^{34}$. Dementsprechend stellen inzwischen auch Ökonomen fest, dass Wachstum »die Arbeitsmarktprobleme nicht lösen « wird ${ }^{35}$.

Auf der Grundlage solcher Analysen müsste nicht nur gesamtgesellschaftlich, sondern auch im Strafvollzug das Verhältnis von Arbeit, Bildung und Freizeit neu bestimmt werden. Natürlich kämen für ihn die bisher hinsichtlich der freien Gesellschaft diskutierten - und ohnehin weitgehend abgelehnten - Lösungsmodelle nicht in Betracht. Er müsste, soweit sinnvolle Arbeit auch bei Ausschöpfung aller seiner Möglichkeiten nicht in hinreichendem Maße zur Verfügung gestellt werden und Berufsausbildung allein keine ausreichende Basis für soziale Integration bieten kann, stärker auf Bildung im umfassenden Sinn des Wortes setzen.

Der Strafvollzug könnte bei einer Neujustierung des Dreiecksverhältnisses von Arbeit, Bildung und Freizeit einerseits an Gedankengänge anknüpfen, die bereits im Rahmen der Strafvollzugsreform wenngleich unter gänzlich andersgearteten gesellschaftlichen und wirtschaftlichen Voraussetzungen - entwickelt worden sind, andererseits auf praktische Erfahrungen rekurrieren, wie sie allgemein im Bildungsbereich gemacht werden können. Bereits 1971 sind »Arbeit und Erwachsenenbildung « in einem zugegebenermaßen recht anspruchsvollen Sinne als Eckpfeiler eines Strafvollzugs begriffen worden, der Teil des gesellschaftlichen Bildungssystems werden sollte. In programmatischer Weise ist damals die sozial integrierende und zukunftsorientierte Bedeutung der Bildung auf den Nenner einer »éducation permanente« gebracht, ihr »berufs- und lebensbegleitende Funktion « mit der Begründung zugeschrieben worden: »Denn wird der rasche Wandel nicht durch eine ihn begleitende Bildung in einem ständigen Lernprozeß verarbeitet, so wird die Gesellschaft ,falsch programmiert und der Einzelne wird zum Spielball unbegriffener Mächte. « ${ }^{36}$ Das sind Feststellungen, die sich heute angesichts einer verbreiteten gesellschaftlichen Verunsicherung, wenn nicht Orientierungslosigkeit wie Prognosen lesen, die inzwischen durch die Realität eingeholt worden sind. Inzwischen haben sich freilich im Verhältnis der beiden gesellschaftlichen Koordinaten die Gewichte kräftig verschoben. Nunmehr dürfte die Bildung im Verhältnis zur Arbeit für das Leben des Einzelnen wie der Gesellschaft im Ganzen noch mehr an Bedeutung gewonnen haben. Aber immerhin belegen neuere Studien namentlich die Wirksamkeit beruflicher Weiterbildung - wenngleich es auch hier, wie die Praxis zeigt, einen langen Atem braucht ${ }^{37}$.

Einer besonderen Begründung bedürfte also ein entsprechender Wandel der Vollzugsstrategie nicht. Zum einen hat sich Bildung längst zu jenem Eckpfeiler im Lebenslauf herauskristallisiert, von dem die Zukunft eines Menschen - in starkem Maße, wenn auch keineswegs allein - abhängt. Das gilt schlechterdings für jedermann, ob er nun freier Bürger oder in Haft ist - im letzteren Falle in wohl noch akzentuierterer Weise. Zum anderen ist Arbeitslosigkeit hinter Mauern namentlich für Erwachsene - die sich gar noch überwiegend im besonders leistungsfähigen Lebensalter befinden geradezu Gift, sowohl was die gegenwärtige als auch die zukünftige Lebensgestaltung betrifft. Wenn sich ein zumindest gleichwertiges Äquivalent für fehlende - Arbeit anbietet, dann ist es die Entwicklung und Förderung individueller Fähigkeiten und Eigenschaften, welche die ohnehin begrenzten Chancen einer Reintegration erhöhen können.

Der Autor ist emeritierter Hochschullehrer und Mitverfasser des Standard-Kommentars zum StVollzG.

\section{Fußnoten}

1 Vgl. z. B. Kruis, ZRP 20001, 1; Wagner, ZRP 2000, 169; Feest (Hrsg.), AK-StVollzG, 4. A. 2000, Rn. 11 vor § 1; Bonk, JZ 2000, 435; Gusy / Lührmann, StV 2001, 46; Lange, DÖV 2001, 898; Kulas, Privatisierung hoheitlicher Verwaltung - Zur Zulässigkeit priavter Strafvollzugsanstalten, 2. A. 2001; Stober (Hrsg.), Privatisierung im Strafvollzug?, 2001; Wohlgemuth, in: Wischka u. a. (Hrsg.), Justizvollzug in neuen Grenzen. Modelle in Deutschland und Europa, 2002, 58; Arloth/Lückemann, StVolzG, 2004, § 155 Rn. 2; Kammeier, in: Streben nach Gerechtigkeit. FS Tondorf, 2004, 61, 74 ff.; Calliess / Müller-Dietz, StVollzG, 1o. A. 2005, Einl Rn. 45, § 155 Rn. 2-5; Schwind/Böhm/Jehle, StVollzG, 4. A. 2005, § 155 Rn. 1; Kirsch, KrimJ 2005, 128; Müther, in: Herrfahrdt (Hrsg.), Privatisierung des Haftvollzuges und Kriminalpolitik in Europa, 2005, 11.

2 Vgl. z. B. J. R. Saul, Der Markt frißt seine Kinder. Wider die Ökonomisierung der Gesellschaft, 1998; V. Forrester, Der Terror der Ökonomie, 1998; D. Kurbjuweit, Die Diktatur der Ökonomie und ihre Folgen, 2003; R. Sennett, Die Kultur des neuen Kapitalismus, 2005.

3 Sack, in: Reindl (Hrsg.), Effektivität, Effizienz und Ethik in Straffälligenhilfe und Kriminalpolitik, 1998, 87; Feest / Lesting, AK-StVollzG, Rn. 11 vor § 1; Walter, in: FS Müller-Dietz, 2001, 961; Brüchert, in: Nickolai / Reindl (Hrsg.), Sozialer Ausschluss durch Einschluss, 2001, 51; Schellhoss, NK 1/2004, 8. Zur »Kommerzialisierung der Sicherheit« auch Markantonatou, Der Modernisierungsprozess staatlicher Sozialkontrolle. Aspekte einer politischen Kriminologie, 2005, $165 \mathrm{ff}$.

4 Nibbeling, die Privatisierung des Haftvollzugs. Die neue Gefängnisfrage am Beispiel der USA, 2001; Bosch / Reichert, ZStW 113 (2001), 207; Smartt, ZfStrVo 2001, 67; dies., NK 4/2001, 8; Kaiser, FS Trechsel, 2002, 869; Giefers-Wieland, Private Strafvollzugsanstalten in den USA - eine Perspektive für Deutschland?, 2002.

5 K. Kruis, ZRP 2000, 1; Ch. Gusy, in: Stober (Fn. 1), 5, 16 ff.; Gusy / Lührmann, StV 2001, 46, 51; Kammeier (Fn. 13), 74; Calliess / Müller-Dietz (Fn. 26), § 155, Rn. 2; 
Schwind / Böhm / Jehle (Fn. 26), § 155 Rn. 1; Müther (Fn. 1), 15.

6 Gusy / Lührmann, StV 2001, 49, 54; Kammeier (Fn. 1),

74 (m. w. Nachw.); Calliess / Müller-Dietz, § 155 Rn. 2.

7 BVerfGE 35, 202, 235; 98, 169, 200.

8 Kammeier (Fn. 1), 75; Calliess / Müller-Dietz, § 155 Rn. $6 \mathrm{f}$.

9 Kulas, in: Stober (Fn. 1), 35, $40 \mathrm{ff}$.

10 Bonk, JZ 2000, 435, 442; Ch. Wagner, ZRP 2000, 169, 172; Wohlgemuth, KrimPäd 2000, 89 f.

11 Vgl. Fn. 7.

12 Gusy / Lührmann, StV 2001, 49, 54; Calliess / MüllerDietz, § 155 Rn. 3, 5; Schwind / Böhm/Jehle, § 155 Rn. 1.

13 Calliess / Müller-Dietz, Einl Rn. 31, § 155 Rn. 5.

14 Arloth/Lückemann, § 155 Rn. 4; Calliess / MüllerDietz, $\S 155$ Rn. 4.

15 Arloth / Lückemann, § 155 Rn. 4.

16 Vgl. etwa Flügge / Maelicke / Preusker (Hrsg.), Das Gefängnis als lernende Organisation, 2001; Wischka u. a. (Fn. 1); Calliess / Müller-Dietz, Einl Rn. 45, § 155 Rn. 2. 17 Arloth/Lückemann, § 155 Rn. 2.

18 BVerfGE 40, 285. Vgl. Calliess / Müller-Dietz, Einl Rn. $34 \mathrm{f}$.

19 Gusy / Lührmann, StV 2001, 48, 54; Calliess / MüllerDietz, § 155 Rn. 9.
20 Calliess / Müller-Dietz, § 155 Rn. 7.

21 Calliess / Müller-Dietz, § 155 Rn. 9.

22 Calliess / Müller-Dietz, § 155 Rn. 10.

23 In der neuen Justizvollzugsanstalt Hünfeld obliegen dem privaten Unternehmen u. a. »die sozialarbeiterische, psychologische und pädagogische Betreuung der Gefangenen, die Beratungsleistungen für die Gefangenen (Drogen-, Ausländer-, Schuldnerberatung), die Organisation und Durchführung der Freizeitveranstaltungen für die Gefangenen, insbesondere der Gefangenensport « (Presseinformationen des Hessischen Ministeriums der Justiz vom 12.12.2004, 2. und 15.11.2005; Kirsch, KrimJ $2005,128,137 \mathrm{f}$.

24 So hat der private Unternehmer in der Justizvollzugsanstalt Hünfeld verschiedene Versorgungsleistungen etwa den Betrieb der Anstaltsküche und die Versorgung der Gefangenen mit Verpflegung - zu erbringen (vgl. Fn. 23).

25Calliess / Müller-Dietz, § 155 Rn. 3 a.E.

26 Calliess / Müller-Dietz, § 155 Rn. 11.

27 JVB-Presse, Nov. 2005, 2 ff., 3; Straubinger Tagblatt vom 13.10.2005.

28 H. Cornel, ZfStrVo 2005, 48; Müller-Dietz, ZfStrVo 2005, 38; ders., ZRP 2005, 156.
29 ZfStrVo 2005, 366. Über entsprechende Auswirkungen einer solchen Verfassungsänderung vgl. z. B. die Presseinformation vom 18.11.2005 aus dem Hamburger Senat, wonach Justizsenator Kusch »die Sicherheit als Vollzugsziel Nummer 1 1 gesetzlich festlegen will.

30 In diese Richtung geht z.B. die Kritik Käppners, SZ vom 8.11.2005, S. 4: »Im Strafvollzug etwa geistern abenteuerliche Privatisierungsideen herum, bei denen es vor allem ums Geld geht und weniger um das Recht. «

31 Vgl. z. B. Südwest-Presse vom 27.4.2005; Ruhrnachrichten vom 18.8.2005; NZZ vom 29 9.2005.

32 Vgl. z. B. Allgäuer Zeitung vom 30.4.2005; Badische Zeitung vom 26.9.2005; IHK Wirtschaft im Südwesten 11/2005, 42. Vgl. auch Fn. 16 und 31.

33 Z.B. Rifkin, Das Ende der Arbeit und ihre Zukunft, 1995; Beck, Die Zukunft von Arbeit und Demokratie, 1999.

34 Assheuer, Die Zeit v. 20.10.2005, 49.

35 FAZ vom 19.11.2005, S. 12.

36 Etscheit, Die Zeit vom 24.11.2005, S. 87.

\section{Privatisierung von Strafvollstreckung am Beispiel der Vermeidung von Ersatzfreiheitsstrafen durch Gemeinnützige Arbeit in Bayern}

Gabriele Kawamura-Reindl

W ährend die kontroverse Diskussion des Themas Privatisierung auch Einzug in die Strafjustiz gehalten hat gibt es in der Bundesrepublik seit Jahren viele konkrete Beispiele für eine Privatisierung von Maßnahmen der Strafvollstreckung durch Übertragung an Freie Träger: So ist zu denken an ambulante Maßnahmen nach dem JGG, deren Durchführung in großem Umfang durch Freie Träger der Jugendhilfe erfolgt, an den Täter-Opfer-Ausgleich, der auch im Bereich des Allgemeinen Strafrechts u. a. von Trägern der Freien Wohlfahrtspflege umsetzt wird, an professionelle Haftentlassenenhilfe in privater Trägerschaft, an die Gemeinnützige Arbeit zur Abwendung von Ersatzfreiheitsstrafen durch die Freie Straffälligenhilfe, an die Vereine Chance e.V. und Prisma e.V. in Baden-Württemberg, die Teile des Jugendstrafvollzuges übernehmen, an den österreichischen Verein Neustart e.V., dem in Teilen Baden-Württembergs Aufgaben der Bewährungshilfe übertragen wurden und die Übertragung des sog. Betreuungsmanagements auf einen privaten Träger in der teilprivatisierten hessischen JVA Hünfeld durch den britische Konzern Serco $\mathrm{GmbH}$, um nur einige zu nennen. Bei genauerer Betrachtung unterscheiden sich diese Privatisierungsmaßnahmen deutlich danach, wer Träger ist (Non-Profit vs. Profit-Unternehmen), wer sie iniitiert hat (Kriminalpolitik von unten vs. Beauftragung eines Privaten Trägers durch die Jus- tiz), welche Ziele sie verfolgen, welche konkreten Maßnahmen sie durchführen, wer die Kosten trägt und über welche (über) Handlungsspielräume sie verfügen, um nur einige Dimensionen zu nennen.

Privatisierung von Aufgaben Sozialer Arbeit im Rahmen der Strafvollstreckung ist also auch in Deutschland nicht neu, sondern vielmehr eine Art von Sammelkategorie für das Outscourcing von Maßnahmen, die die Justiz anordnet, die aber von privaten Trägern umgesetzt werden. Die zunehmende Finanzknappheit der öffentlichen Hand hat dazu geführt, dass die Grundsätze der Wirtschaftlichkeit und Sparsamkeit zunehmend zu einer Prüfung der Frage führen, inwieweit staatliche Aufgaben durch Ausgliederung und Entstaatlichung oder Privatisierung erfüllt werden können. In vielen Bereichen öffentlicher Verwaltung wird zur Konkretisierung dieser Privatisierungsmaßnahmen zunehmend der Begriff »Public Private Partnership benutzt. »Public Private Partnership (PPP) bezeichnet das partnerschaftliche Zusammenwirken von öffentlicher Hand und Privatwirtschaft mit dem Ziel einer besseren wirtschaftlichen Erfüllung öffentlicher Aufgaben als bisher. PPP-Projekte erfassen das gesamte Spektrum zwischen der rein hoheitlichen Realisierung öffentlicher Aufgaben einerseits und der vollständigen Privatisierung öffentlicher Aufgaben andererseits. « (Bertelsmann Stif- tung u.a. 2005). Unter den Oberbegriff PPP lassen sich unterschiedlichste Sachverhalte und Erscheinungsformen subsumieren, die von formaler Privatisierung (= Organisationsprivatisierung), Teilprivatisierung, Vollprivatisierung, materieller Privatisierung (= Aufgabenprivatisierung) bis zur funktionalen Privatisierung (= Contracting out) reichen. Bei der Privatisierung von Aufgaben Sozialer Arbeit im Rahmen der Strafvollstreckung dürften wir es v. a. mit letzteren beiden Formen zu tun haben. In den meisten Fällen liegt dem $\mathrm{Zu}$ sammenwirken zwischen öffentlicher Verwaltung und privaten Trägern in der Regel eine vertragliche Regelung zugrunde, die gegenseitige Rechte und Pflichten, Laufzeiten, Leistungen, Vergütungen und andere Konditionen festschreibt. Hiervon ist die Straffälligenhilfe in einigen Bereichen der Privatisierung, z. B. dem Täter-Opfer-Ausgleich oder der Strafentlassenenhilfe noch relativ weit entfernt. Dies betrifft v. a. die Laufzeiten (eine auf ein Jahr festgelegte und damit ungesicherte Finanzierung darf inzwischen als die Regel und nicht als die Ausnahme betrachtet werden und stellt keine tragfähige Basis für längerfristige Planungen dar), aber auch die vertragliche Festschreibung von Leistungen und Vergütungen. Nicht nur in der Straffälligenhilfe verlagert der Staat durch Privatisierungen finanzielle Risiken auf private Träger, die bei einer absehbaren Deckelung zuvor budgetierter 